\title{
Design of Complex Network Theory Experiment Platform Based on Matlab
}

\author{
Zou Qingyu* \\ College of Electrical and Information Engineering \\ Beihua University \\ Jilin, China \\ zouqingyu2002@126.com
}

\author{
Ruan Haitao \\ College of Electrical and Information Engineering \\ Beihua University \\ Jilin, China \\ 2434648311@qq.com
}

\author{
Wang Tao \\ College of Electrical and Information Engineering \\ Beihua University \\ Jilin, China \\ wt1997123@foxmail.com
}

\begin{abstract}
Complex network as a new research direction has gradually become a hot topic in the various fields of science, and the relevant research results continue to emerge. In order to analyze the structural characteristics of complex networks more efficiently, all kinds of analysis software, package and experimental platform are emerging. However, there are gaps in the identification of overlapping modules in complex directional network. In this paper, we design a DNMPQEP platform with overlapping module identification function and topological structure attribute analysis function. The platform is based on Mtalab software, including a variety of overlapping module recognition algorithm in directional network.
\end{abstract}

Keywords-complex network; matlab; experiment platform; structural

\section{INTRODUCTION}

Since the scale-free and small worlds of the network have been discovered, complex networks have become the research hotspots in various fields of science, and the related scientific research achievements have been emerging[1]. In order to analyse the structural characteristics of complex networks more efficiently, various types of analysis software, packages and experimental platforms are emerging[2-6]. Most of this software has visualization, topology analysis, node clustering, pattern recognition and other functions. However, there are gaps or shortcomings in the evaluation of the overlapping network module and the evaluation of the quality of the module division. In this paper, an experimental platform DNIMQEP with topology attribute analysis, overlapping module identification and module identification quality evaluation function is designed for directional network with overlapping module structure. DNIMQEP based on Matlab software, using human-computer interaction interface. MATLAB is a high-tech computing environment for scientific computing, visualization and interactive programming designed by the United States

This work is supported partially by the Jilin Province Science and Technology Development Project (Grant NO.20170520057JH), partially by the Beihua University Dr. Scientific Research Fund (Grant NO. 2015557) partially by the Jilin Province department of education Science and Technology Plan Projects. (Grant NO 201658, Grant NO. 201659). math works company. Its basic data unit is a matrix. It integrates numerical analysis, matrix computing, scientific data visualization, and modeling and simulation of nonlinear dynamic systems into an easy-to-use window environment. Matlab is mainly used in engineering computing, control design, signal processing and communication, image processing, signal detection, financial modeling design and analysis and other fields, for scientific research, engineering design and many scientific fields, which must be effective numerical calculation, to provide a Comprehensive solution.

\section{The Function And Structure Of The Platform}

DNIMQEP modular design, including network topology attribute analysis and overlapping modules to identify two functional classes. Topological attribute analysis includes the calculation of the basic topology attributes of the network, the calculation of the degree distribution, the cumulative distribution, the degree distribution, the cumulative degree distribution, the closeness distribution and the local clustering coefficient distribution. The overlapping module recognition function mainly uses the correlation algorithm to identify the complex directional network with overlapping module structure and uses the module recognition evaluation function to measure the quality of the recognition result.

DNIMQEP using human-computer interaction interface, the main interface shown in Fig. 1, divided into two parts of the menu bar and display area. The main interface can calculate and display the basic topology properties of the network, such as the number of nodes, the number of edges, the mean value, and the mean value of the input network. Through the main interface menu bar in the "network structure attribute distribution" option can open the "network structure distribution comparison" interface, the interface shown in Fig. 2. This interface can compare the distribution of attributes for two different networks. The two attributes are displayed by the "Network Import 1" and "Network Import 2" options. The topology attribute distribution curves are displayed by two 
windows: "Network Structure Attribute Distribution Curve 1" and "Network Structure Attribute Distribution Curve 2". Through the "network structure attribute" option, we can calculate the distribution of different network structure attributes, including: degree distribution, degree distribution, cumulative distribution, cumulative distribution, shortest path distribution, cumulative shortest path distribution, local clustering coefficient distribution, the cumulative clustering coefficient distribution, the number distribution and the cumulative distribution of the distribution.

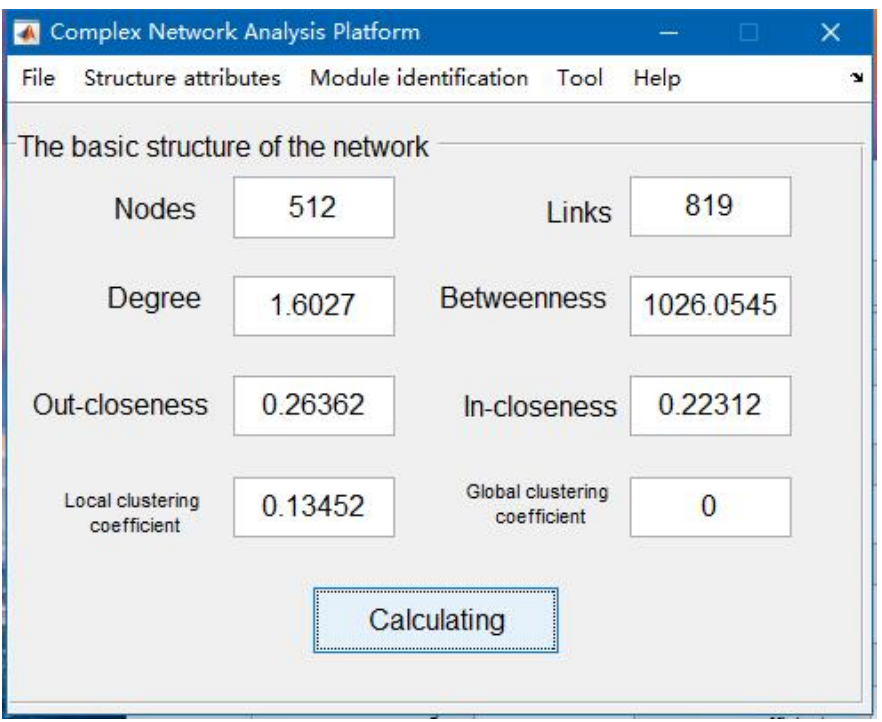

Fig. 1. The main interface of DNIMQEP.

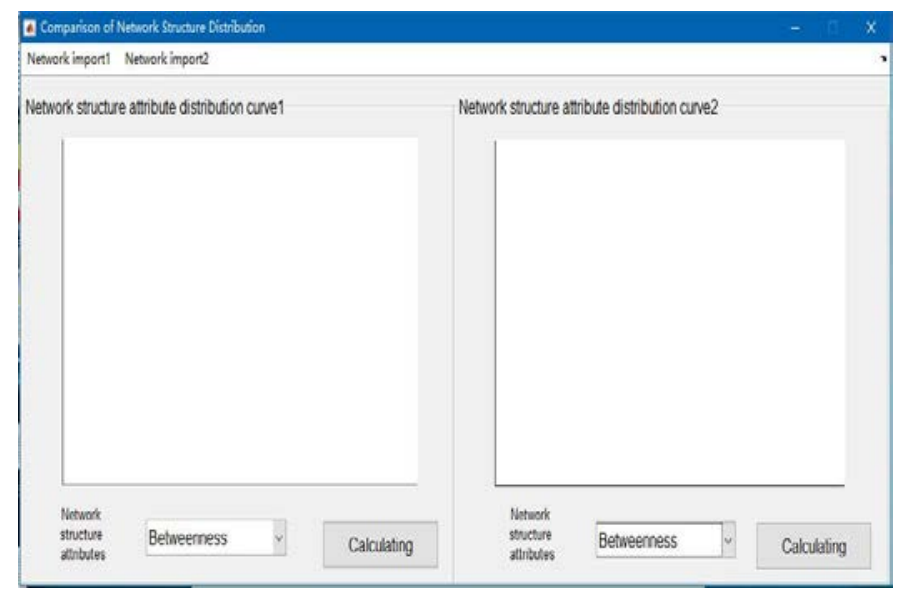

Fig. 2. The interface of network structure distribution comparison.

Complex network module identification function interface shown in Fig. 3. Enter the network adjacency matrix through the "Network Import" option in the menu bar and select the algorithm for identifying the module via the "Module recognition algorithm" option. The module recognition results are displayed by the table. The table can not only show the number of modules, but also can display the number of nodes in each module, the number of sides, the average value of the basic topology of the property value. "Module degree" is used to evaluate the quality of module identification. This option can use the module recognition evaluation function to score the results of the module division, and finally get the value of the module to identify the quality of identification.

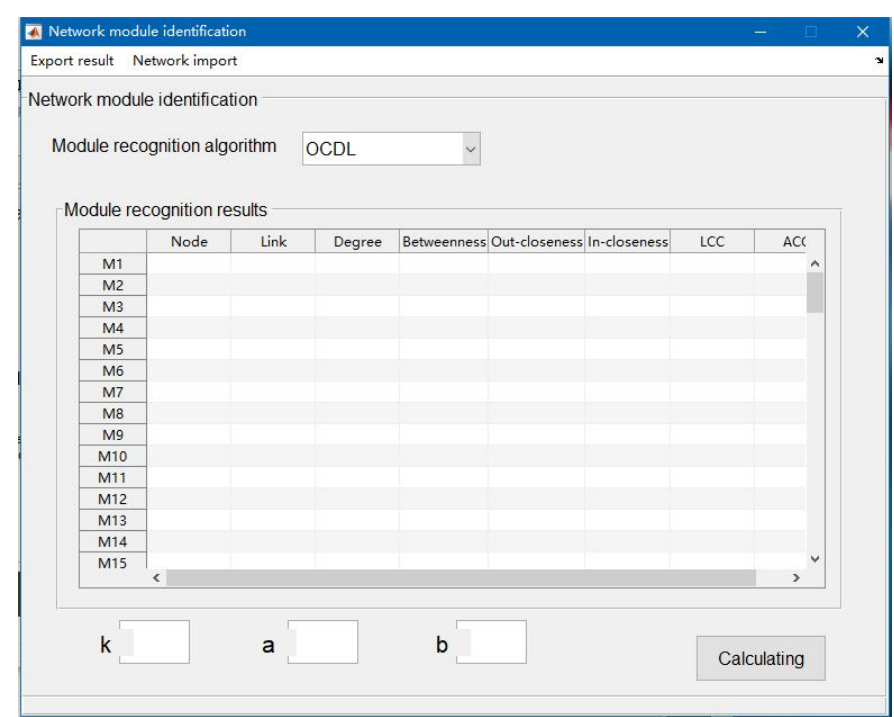

Fig. 3. The interface of network structure distribution comparison.

\section{NETWORK MODUlE RECOGNITION EVALUATION FUNCTION}

Modularity is an important attribute of complex networks. In recent years, a large number of network module recognition methods have been proposed, but there is still no universally accepted module definition. Most module identification methods divide the network modules according to their definition of modules. In order to evaluate the quality of the module division, Newman et al. proposed a quality evaluation function called the modularity Q [7]. The modularity Q function calculates the degree of the module by comparing the distribution of the edges in the actual network with the random network. In the undirected network with n nodes and m edges, the degrees of nodes $\mathrm{i}$ and $\mathrm{j}$ are ki and kj, respectively. The modularity Q is.

$$
Q=\left(\sum_{i j}\left(A_{i j}-k_{i} k_{j} / 2 m\right) \delta\left(C_{i}, C_{j}\right)\right) / 2 m
$$

Where $\mathrm{A}$ is the adjacency matrix of the network. When points $\mathrm{i}$ and $\mathrm{j}$ in the same module, its value is 1 , otherwise 0 .

\section{ACTUAL NETWORK APPLICATION COMPARISON}

We have analysis the e-mail network and identify the modules from it with DNIMQEP experimental platform. Email is a common way for people to send information. The sending and receiving process of mail forms a communication network, also called E-mail network. When the e-mail is transmitted, the sender and receiver's address is recorded in the server at the same time. We build an e-mail network, which the sender of the server and the recipient's address as a node, and the e-mail between them as an edge. This paper analyzes and calculates the e-mail network of the University of Virginia. The network contains 1669 users, including researchers, teachers, managers, skilled workers and graduate students. After deleting 
the sender with more than 50 sending and giving each edge a random direction, the network contains 1133 nodes and 5452 edges. Specific topology information shown in Table 1 . Where Node represents the number of nodes in the network, Link represents the number of edges in the network, Dg represents the node degree average, Ap represents the node proximity average, Cc represents the node local clustering coefficient average[ 8,9$]$. We identify the modules of e-mail network, the results shown in Table 2, the e-mail network is divided into six modules, represented by M1, M2, M3, M4, M5, M6 in Table 2.

TABLE 1. TOPOLOGY E-MAIL NETWORK.

\begin{tabular}{|c|c|c|c|c|c|}
\hline Node & Link & Dg & Ap & Cc & Bn \\
\hline 1133 & 5451 & 7.16 & 0.43 & 0.148 & 794.5 \\
\hline
\end{tabular}

TABLE 2. THE RESULT OF E-MAIL NETWORK MODULE IDENTIFICATION.

\begin{tabular}{|c|c|c|c|c|c|c|c|}
\hline $\mathbf{M}$ & $\mathbf{N}$ & Link & Dg & Ap & Bn & Cc & Ccg \\
\hline M1 & 58 & 135 & 3.65 & 0.85 & 6.35 & 0.31 & 0.27 \\
\hline M2 & 120 & 324 & 4.10 & 0.60 & 66.06 & 0.24 & 0.18 \\
\hline M3 & 55 & 145 & 3.91 & 0.81 & 7.20 & 0.30 & 0.24 \\
\hline M4 & 85 & 200 & 3.92 & 0.67 & 38.63 & 0.28 & 0.20 \\
\hline M5 & 933 & 5175 & 7.38 & 0.43 & 700.7 & 0.15 & 0.07 \\
\hline M6 & 562 & 1270 & 3.93 & 0.54 & 172.9 & 0.15 & 0.09 \\
\hline
\end{tabular}

\section{SUMMARY}

Based on the Mtalab software, a complex network structure analysis experiment platform DNIMQEP with module recognition function and topology attribute analysis function is designed and implemented by using the network module recognition algorithm. The platform uses the human - computer interaction interface, the operation is simple, the result is clear, and embeds a module identification quality evaluation function Q which only applies to the module overlapping directional network. We use the DNIMQEP test platform to analyze the email network; the results show that the platform can effectively identify modules in complex networks.

\section{ACKNOWLEDGMENT}

This work is supported partially by the Jilin Province Science and Technology Development Project (Grant NO.20170520057JH), partially by the Beihua University Dr. Scientific Research Fund (Grant NO. 2015557), partially by the Jilin Province department of education Science and Technology Plan Projects. (Grant NO 201658, Grant NO. 201659).

\section{REFERENCES}

[1] S. Boccaletti, R. Criado, and M. Romance, "COMPLEX NETWORKS", International Journal of Bifurcation and Chaos, vol. 19, pp. 661-663, Feb 2009.

[2] N. Yosef et al., "ANAT: A Tool for Constructing and Analyzing Functional Protein Networks", Science Signaling, vol. 4, Oct 2011.

[3] M. Kohl, S. Wiese, and B. Warscheid, "Cytoscape: software for visualization and analysis of biological networks", (in eng), Methods Mol Biol, vol. 696, pp. 291-303, 2011.

[4] A. T. Kalinka and P. Tomancak, "linkcomm: an R package for the generation, visualization, and analysis of link communities in networks of arbitrary size and type", (in eng), Bioinformatics, vol. 27, pp. 2011-2, Jul 152011.

[5] S. Ghosh, Y. Matsuoka, Y. Asai, K. Y. Hsin, and H. Kitano, "Software for systems biology: from tools to integrated platforms", Nature Reviews Genetics, vol. 12, pp. 821-832, Dec 2011.

[6] I. Drozdov, C. A. Ouzounis, A. M. Shah, and S. Tsoka, "Functional Genomics Assistant (FUGA): a toolbox for the analysis of complex biological networks", (in eng), BMC Res Notes, vol. 4, p. 462, 2011.

[7] M. E. J. Newman, "Modularity and community structure in networks", (in English), Proceedings of the National Academy of Sciences of the United States of America, vol. 103, pp. 8577-8582, Jun 62006.

[8] M. E. J. Newman, "Communities, modules and large-scale structure in networks", Nature Physics, vol. 8, pp. 25-31, Jan 2012.

[9] M.E.J.Newmen, Networks: An Introduction. New York: Oxford University Press, 2010. 